\title{
Quantum Annihilation of Anti-de Sitter Universe
}

\author{
Iver Brevik \\ Division of Applied Mechanics \\ Norwegian University of Science and Technology \\ N-7491 Trondheim, Norway \\ Sergei D. Odintsov \\ Tomsk State Pedagogical University \\ 634041 Tomsk, Russia
}

September 26, 2021

\begin{abstract}
We discuss the role of conformal matter quantum effects (using large $N$ anomaly induced effective action) to creation-annihilation of an Anti-de Sitter Universe. The arbitrary GUT with conformally invariant content of fields is considered. On a purely gravitational (supersymmetric) AdS background, the quantum effects act against an (already existing) AdS Universe. The annihilation of such a Universe occurs, what is common for any conformal matter theory. On a dilaton-gravitational background, where there is dilatonic contribution to the induced effective action, the quantum creation of an AdS Universe is possible assuming fine-tuning of the dilaton.
\end{abstract}

1. There is a large interest now in studies related to Anti-de Sitter (AdS) backgrounds. This is caused by several reasons. First of all, via the AdS/CFT correspondence (for a review,see [1]) investigating the classical IIB supergravity on an AdS background (after compactification), one can get answers for the dual (boundary) quantum gauge theory. Second, the AdS space is an extremely symmetric one (maximum number of Killing vectors), like Minkowski space. Moreover, it is a well-known supersymmetric background for supergravity theories. For strings the backgrounds with AdS section are often suspected to be the exact vacuum state. Third, according to some cosmological data the inflationary Universe could have the spatial section with negative curvature. Generalizing this for a brane world one can speculate on the possibility of $4 \mathrm{~d}$ AdS stage (or regions) in the early Universe.

The important question is: how should one create the AdS regions in the early Universe? The mechanisms of such creation maybe important for the realization of AdS Black Holes presence. In this note we investigate the role of matter quantum effects to the Anti-de Sitter Universe. It is well known that the inflationary (de Sitter) Universe maybe created completely by quantum effects of conformally invariant matter. On the contrary, as we will show below, the quantum creation of Anti-de Sitter Universe by quantum effects is rather unrealistic. The quantum annihilation of such a Universe occurs. Only in presence of dilaton the fine-tuning of the dilaton solution may lead to quantum creation of the AdS Universe (an example of quantum maximally supersymmetric YM theory conformally coupled to conformal background supergravity). 
2. Let us start from one form of the metric describing four-dimensional Anti-de Sitter $\left(\mathrm{AdS}_{4}\right)$ spacetime,

$$
\mathrm{d} s^{2}=\mathrm{e}^{-2 \lambda \widetilde{x}_{3}}\left(\mathrm{~d} t^{2}-\left(\mathrm{d} x^{1}\right)^{2}-\left(\mathrm{d} x^{2}\right)^{2}\right)-\left(\mathrm{d} \widetilde{x}^{3}\right)^{2},
$$

having a negative effective cosmological constant $\Lambda=-\lambda^{2}$. One may present this metric in conformally flat form via the transformation

$$
y=x^{3}=\frac{\mathrm{e}^{\lambda \widetilde{x}_{3}}}{\lambda} .
$$

Then

$$
\mathrm{d} s^{2}=a^{2}\left(\mathrm{~d} t^{2}-\mathrm{d} \boldsymbol{x}^{2}\right)=a^{2} \eta_{\mu \nu} \mathrm{d} x^{\mu} \mathrm{d} x^{\nu},
$$

with $a=\mathrm{e}^{-\lambda \widetilde{x}^{3}}=1 /\left(\lambda x^{3}\right)=1 /(\lambda y)$. This form of the metric is often useful in the study of quantum gauge theory via the SG dual.

Imagine now that the early Universe is described by some quantum grand unified theory (GUT) containing $N_{s}$ conformal scalars, $N_{f}$ spinors and $N_{v}$ vectors. It is enough to consider only free fields in such a GUT as radiative corrections are not important for our purposes. Moreover, the large class of GUTs - asymptotically finite and asymptotically conformal GUTs (see the book [2] for a review) - maybe presented as a collection of free fields at strong curvature (in the early Universe). Unlike to the de Sitter space the AdS space is supersymmetric background of GUT if it is SUSY. Note that quantum fields on negative curvature space have been reviewed in ref.[3].

The quantum GUT under consideration produces the well-known conformal anomaly (for a review see 顿)

$$
T=b\left(F+\frac{2}{3} \square R\right)+b_{1} G+b_{2} \square R
$$

where $b$ and $b_{1}$ are constants,

$$
b=\frac{N_{s}+6 N_{f}+12 N_{v}}{120(4 \pi)^{2}}, \quad b_{1}=-\frac{N_{s}+11 N_{f}+62 N_{v}}{360(4 \pi)^{2}},
$$

while $F$ is the square of the Weyl tensor,

$$
F=R_{\mu \nu \alpha \beta} R^{\mu \nu \alpha \beta}-2 R_{\mu \nu} R^{\mu \nu}+\frac{1}{3} R^{2}
$$

and $G$ is the Gauss-Bonnet invariant. Note that the constant $b_{2}$ is known to be in general ambiguous, as it may be changed by a finite renormalization of the gravitational action. In the following we put $b_{2}=0$, since this does not influence the physical consequences. The features of a particular GUT are encoded in the numerical values of $b$ and $b_{1}$ as their signs (what is important for us) do not change.

Using the above conformal anomaly one can easily calculate the anomaly-induced effective action [5]. Having in mind the applications for quantum induced AdS space we consider a metric similar to (3), i.e. $g_{\mu \nu}=\mathrm{e}^{2 \sigma(y)} \eta_{\mu \nu}$, where $\eta_{\mu \nu}$ is the Minkowski metric. Then the 
techniques of ref. [5] may easily be applied, and the following anomaly-induced effective action is obtained,

$$
W=\int \mathrm{d}^{4} x\left[2 b_{1} \sigma \square^{2} \sigma-2\left(b+b_{1}\right)\left(\square \sigma+\eta^{\mu \nu}\left(\partial_{\mu} \sigma\right)\left(\partial_{\nu} \sigma\right)\right)^{2}\right] .
$$

Since $\sigma$ is assumed to depend only on $y$, this expression may be simplified:

$$
W=V_{3} \int \mathrm{d} y\left[2 b_{1} \sigma \sigma^{\prime \prime \prime \prime}-2\left(b+b_{1}\right)\left(\sigma^{\prime \prime}+\left(\sigma^{\prime}\right)^{2}\right)^{2}\right] .
$$

Here $\sigma^{\prime}=\mathrm{d} \sigma / \mathrm{d} y$. Formally, this expression coincides with the effective action on a time dependent conformally flat background, but its physics is of course different. One should also remember that the total effective action consists of $W$ plus some conformally invariant functional. In the case of a conformally flat background, as considered here, this conformally invariant functional is a non-essential constant. Only if one considers periodicity on some of the coordinates (say, AdS BH) will this constant become more important, as a kind of Casimir energy, since it will depend on the radius of the compact dimension.

In order to take into account the quantum matter effects in the AdS Universe we should add the anomaly-induced action to the classical gravitational action

$$
S_{\mathrm{cl}}=-\frac{1}{\kappa} \int \mathrm{d}^{4} x \sqrt{-g}(R+6 \Lambda)=-\frac{1}{\kappa} \int \mathrm{d}^{4} x \mathrm{e}^{4 \sigma}\left(-6 \mathrm{e}^{-2 \sigma}\left(\left(\sigma^{\prime}\right)^{2}+\left(\sigma^{\prime \prime}\right)\right)+6 \Lambda\right) .
$$

The sum of classical action and quantum effective action describes the dynamics of whole quantum system.

Equations of motion following from the action $S_{\mathrm{cl}}+W$ are

$$
\frac{a^{\prime \prime \prime \prime}}{a}-\frac{4 a^{\prime} a^{\prime \prime \prime}}{a^{2}}-\frac{3\left(a^{\prime \prime}\right)^{2}}{a^{2}}+\left(6-6 \frac{b_{1}}{b}\right) \frac{a^{\prime \prime}\left(a^{\prime}\right)^{2}}{a^{3}}+\frac{6 b_{1}\left(a^{\prime}\right)^{4}}{b a^{4}}-\frac{a}{4 b \kappa}\left(-12 a^{\prime \prime}-24 \Lambda a^{3}\right)=0 .
$$

Here prime means derivative with respect to $y$. One may now look for the special AdS-like solutions of Eq. (10): $a=c / y$. When there are no quantum corrections, there is a solution with $c=1 / \sqrt{-\Lambda}$, in accordance with the AdS metric. When the effective cosmological constant $\Lambda=0$, Eq. (10) reduces to $c^{2}=b_{1} \kappa\left(\right.$ at $a(y)=c / y$ ). However, $c^{2}=b_{1} \kappa$ leads to an imaginary scale factor $a$, because $b_{1}<0$. This indicates that matter corrections alone can not create an Anti-de Sitter Universe. This is in contrast to the possibility of creation of a de Sitter Universe by solely matter quantum effects [6]. Indeed, when scale factor depends only on time the sign of curvature (which is positive) is changing. As a result, $a=c / \eta$ with $c^{2}=-b_{1} \kappa$. In other words, there is always a solution - in form of a quantum created de Sitter Universe! On the contrary, the presence of a negative effective cosmological constant in classical theory is a necessary condition for the existence of an Anti-de Sitter Universe, at least within our scenario.

In the general case, the algebraic equation for $c^{2}$ becomes (assuming $\Lambda<0$ ):

$$
\kappa b_{1}-c^{2}-\Lambda c^{4}=0,
$$

and it has the solutions:

$$
c_{1}^{2}=-\frac{1}{2 \Lambda}\left(1+\sqrt{1+4 \kappa b_{1} \Lambda}\right)
$$


and

$$
c_{2}^{2}=-\frac{1}{2 \Lambda}\left(1-\sqrt{1+4 \kappa b_{1} \Lambda}\right) .
$$

The first solution corresponds to the quantum corrected Anti-de Sitter Universe. Here, starting from some bare (even very small!) negative cosmological constant, we get an Anti-de Sitter Universe with a smaller cosmological constant due to quantum corrections. The quantum corrections act against the existing Anti-de Sitter Universe and make it less stable. This is the mechanism of annihilation of Anti-de Sitter Universe.

The second solution corresponds to the imaginary scale factor since it has $c^{2}<0$.

The complete effective action on the solution $a(y)=c / y$ is

$$
\begin{aligned}
S_{\mathrm{cl}}+W & =V_{3} \int \frac{\mathrm{d} y}{y^{4}}\left[6 b_{1} \ln \left(\frac{c^{2}}{y^{2}}\right)-8\left(b+b_{1}\right)-\frac{6\left(\Lambda c^{4}-c^{2}\right)}{\kappa}\right] \\
& =V_{3} \int \frac{\mathrm{d} y}{y^{4}}\left[6 b_{1} \ln \left(\frac{c^{2}}{y^{2}}\right)-8 b-14 b_{1}+\frac{12 c^{2}}{\kappa}\right] .
\end{aligned}
$$

The dependence of effective action from $c^{2}$ is seen.

Thus, we have shown that quantum corrections in an already existing Anti-de Sitter Universe make it less stable, whereas creation of an Anti-de Sitter Universe by quantum corrections alone is impossible. This is in contrast to the de Sitter Universe, which may be created by quantum corrections alone. It is now interesting to understand the role of other effects in our scenario. As such we consider the presence of dilaton. The related dilatonic terms in action may play the role of effective cosmological constant.

3. As an explicit example of theory where dilaton appears in conformal anomaly we consider $\mathcal{N}=4 \mathrm{SU}(N)$ super YM theory covariantly coupled with the background $\mathcal{N}=4$ conformal supergravity (see [7] for an introduction). The corresponding vector multiplet is $\left(A_{\mu}, \psi_{i}, X_{i j}\right)$. There is also complex scalar (axion and dilaton) in the conformal supergravity multiplet. Note that such a theory is not a realistic one.

On the purely bosonic background the conformal anomaly in such a theory is the following [8]

$$
T=b\left(F+\frac{2}{3} \square R\right)+b_{1} G+b_{2} \square R+C\left[\square \phi^{*} \square \phi-2\left(R^{\mu \nu}-\frac{1}{3} g^{\mu \nu} R\right) \partial_{\mu} \phi^{*} \partial_{\nu} \phi\right] .
$$

The last term, with the constant

$$
C=\frac{N^{2}-1}{(4 \pi)^{2}}
$$

is the contribution of dilaton and axion fields. Note also that in adjoint representation $N_{v}=$ $N^{2}-1, N_{s}=6 N_{v}$ and $N_{f}=2 N_{v}$ in the theory under discussion.

Using the conformal anomaly (15), and following ref. [9], it is again not difficult to construct the anomaly-induced effective action $W$ on the conformally flat background, $g_{\mu \nu}=$ $\mathrm{e}^{2 \sigma} \eta_{\mu \nu}$.

With $\sigma$ and $\phi$ depending only on time, one gets, in terms of the conformal time $\eta$ [9],

$$
W=V_{3} \int \mathrm{d} \eta\left[2 b_{1} \sigma \sigma^{\prime \prime \prime \prime}-2\left(b+b_{1}\right)\left(\sigma^{\prime \prime}+\left(\sigma^{\prime}\right)^{2}\right)+C \sigma \operatorname{Re}\left(\phi^{*} \phi^{\prime \prime \prime \prime}\right)\right],
$$


where $V_{3}$ is three-dimensional volume, and $\sigma^{\prime}=\mathrm{d} \sigma / \mathrm{d} \eta$. As usual, the complete effective action is the sum of two terms: $W$ and some conformally invariant functional $W_{1}$. Since we discuss a conformally flat background, $W_{1}$ can only depend on the complex scalar $\phi$, and has to be constant when $\phi$ is constant. Supposing that $W_{1}$ is a local functional (so that the Schwinger-De Witt expansion may be used), we are left with only one possibility,

$$
W_{1}=V_{3} \int \mathrm{d} \eta \operatorname{Re}\left(\phi^{*} \phi^{\prime \prime \prime \prime}\right) \ln \mu^{2} .
$$

The coefficient of $W_{1}$ depends on the regularization used, since it may be absorbed into the scale $\mu$, by a redefinition. Thus our quantum correction to the classical action is $\Gamma=W+W_{1}$.

The usual choice for the complex scalar $\phi$ is

$$
\phi=\chi+\mathrm{ie}^{-\varphi},
$$

where $\varphi$ is the dilaton, and $\chi$ is the R-R scalar (axion) of type IIB supergravity (or conformal supergravity, as above).

Thus, the final expression for the one-loop effective action of $\mathcal{N}=4$ super Yang-Mills theory coupled to conformal supergravity on a conformally flat background is

$$
\Gamma=V_{3} \int \mathrm{d} \eta\left[2 b_{1} \sigma \sigma^{\prime \prime \prime \prime}-2\left(b+b_{1}\right)\left(\sigma^{\prime \prime}+\left(\sigma^{\prime}\right)^{2}\right)+(C \sigma+A) \operatorname{Re}\left(\phi^{*} \phi^{\prime \prime \prime \prime}\right)\right],
$$

where $A$ is some constant depending on regularization, and $\phi=\chi+\mathrm{ie}^{-\varphi}$. One may consider the case of large $N$, then in the large $N$ expansion the proper quantum gravity corrections to $\Gamma$ will be of next-to-leading order.

There are different choices for the classical gravitational action. For example, one can consider the axion-dilatonic gravity by Gibbons-Green-Perry [10], which describes the bosonic sector of type IIB supergravity,

$$
S_{\mathrm{cl}}=-\frac{1}{\kappa} \int \mathrm{d}^{4} x \sqrt{-g}\left(R+\frac{1}{2} g^{\mu \nu} \partial_{\mu} \varphi \partial_{\mu} \varphi+\frac{1}{2} \mathrm{e}^{2 \varphi} g^{\mu \nu} \partial_{\mu} \chi \partial_{\mu} \chi\right) .
$$

In the absence of the dilaton and axion, this reduces to the standard action of general relativity. Using the above choice of a conformally flat metric one may simplify Eq. (20) as follows,

$$
S_{\mathrm{cl}}=-\frac{1}{\kappa} V_{3} \int \mathrm{d} \eta\left(6 \mathrm{e}^{2 \sigma}\left(\left(\sigma^{\prime}\right)^{2}+\left(\sigma^{\prime \prime}\right)\right)+\frac{1}{2} \mathrm{e}^{2 \sigma}\left(\varphi^{\prime}\right)^{2}+\frac{1}{2} \mathrm{e}^{2 \sigma+2 \varphi}\left(\chi^{\prime}\right)^{2}\right) .
$$

Now it is convenient to transform to cosmological time $t$, such that $\mathrm{d} t=a(\eta) \mathrm{d} \eta=\mathrm{e}^{\sigma(\eta)} \mathrm{d} \eta$. The equations of motion may be obtained by variations with respect to $a, \varphi$ and $\chi$.

We will consider the simpler choice when the axion is equal to zero, and the kinetic term for the dilaton in the classical action is absent. The regularization where $A=0$ is also chosen. As a result one comes to the model discussed in ref. [9]. The study of the corresponding effective equations for time-dependent conformally flat metric has been done in the above work. The possibility of quantum creation of de Sitter Universe has been proved. One can give now an analysis related to a quantum corrected Anti-de Sitter Universe in the same way as it was done in previous section. Note, however, that in the case under discussion there is no classical cosmological constant and as a result there is no Anti-de Sitter solution on the classical level. 
The effective equations of motion take the form:

$$
\begin{aligned}
\frac{a^{\prime \prime \prime \prime}}{a}-\frac{4 a^{\prime} a^{\prime \prime \prime}}{a^{2}}-\frac{3 a^{\prime \prime 2}}{a^{2}}+\frac{6 a^{\prime \prime} a^{\prime 2}}{a^{3}}\left(1-\frac{b_{1}}{b}\right)+\frac{6 b_{1} a^{4}}{b a^{4}}+\frac{3 a a^{\prime \prime}}{\kappa b}-\frac{C}{4 b} \varphi \varphi^{\prime \prime \prime \prime} & =0, \\
\ln a \varphi^{\prime \prime \prime \prime}+(\ln a \varphi)^{\prime \prime \prime \prime} & =0 .
\end{aligned}
$$

Here prime means derivative on $y$ and we put $\varphi=\phi$.

Motivated by ref. [9] one can make now the following transformation:

$$
\mathrm{d} z=a(y) \mathrm{d} y .
$$

Then, in terms of the variable $z$ the first of equations (22) is:

$$
a^{2} \dddot{a}+3 a \dot{a} \dddot{a}+a \ddot{a}^{2}-\left(5+\frac{6 b_{1}}{b}\right) \dot{a}^{2} \ddot{a}+\frac{3}{\kappa b}\left(a^{2} \ddot{a}+a \dot{a}^{2}\right)-\frac{C \varphi Y[\varphi, a]}{4 b}=0 .
$$

Here $\dot{a}=\mathrm{d} a / \mathrm{d} z, Y[\varphi, a]$ is given in ref. [9], and the second equation (22) (in terms of $z$ ) is also given in ref. 99] (Eq. (10)). The only difference from the analysis done there, is that the sign of the $1 / \kappa$ term is reversed. Then, as in ref. [9] we search for special solutions

$$
a(z) \simeq a_{0} \mathrm{e}^{H z}, \quad \varphi(z) \simeq \varphi_{0} \mathrm{e}^{-\alpha H z},
$$

Analyzing the second of equations (22) (in terms of $z$ ) and dropping logarithmic term in it (same arguments as in ref. [9] may be given) one comes to the same solution:

$$
\varphi(z)=\varphi_{1} \mathrm{e}^{-\frac{3}{2} H z}+\varphi_{2} \mathrm{e}^{-2.62 H z}+\varphi_{3} \mathrm{e}^{-0.38 H z},
$$

where $\varphi_{1}, \varphi_{2}, \varphi_{3}$ are constants. Substituting the particular solution $\varphi(z)=\varphi_{0} \mathrm{e}^{-\alpha H z}$ in Eq. (24) one obtains:

$$
H^{2} \simeq \frac{1}{\kappa}\left[b_{1}+\frac{C}{24} \varphi_{0}^{2}\left(\alpha^{4}-6 \alpha^{3}+11 \alpha^{2}-6 \alpha\right)\right]^{-1}
$$

The first term in the denominator is always negative, while the second term may be positive only at $\alpha=3 / 2$. Then, it is only with the special dilaton solution $\varphi(z)=\varphi_{1} \mathrm{e}^{-\frac{3}{2} H z}$ (i.e. $\varphi_{2}=\varphi_{3}=0$ ) and at the condition $\varphi_{1}^{2}>12$ one gets the positive $H^{2}$ and, hence, nonimaginary scale factor for AdS Universe. Thus, there occurs the possibility for quantum creation of dilatonic AdS Universe, but it requires a strong fine-tuning of the dilatonic solution. This is, of course, a very non-realistic situation. Note that the corresponding AdS scale factor is:

$$
a(y)=-\frac{1}{H y} .
$$

One can analyze the Eqs. (22) numerically with the same qualitative result; for some very special initial conditions for dilaton and scale factor the quantum creation of AdS Universe is possible. However, in most cases such a process does not occur.

Thus, we demonstrated that quantum effects of conformal matter do not support the creation of an AdS Universe. Moreover, an already existing classical AdS Universe is annihilating due to quantum effects. Only for dilaton-gravitational background (maximally SUSY 
YM theory) where there is dilatonic contribution to conformal anomaly and to induced effective action the quantum effects may create AdS Universe subject to strong fine-tuning.

However, the probability of such creation is much less than the same process for de Sitter Universe at similar conditions.

The model under discussion maybe understood also as a simplified model for creationannihilation of AdS Black Hole. Taking additional contribution to effective action due to geometrical structure of AdS BH one can repeat this analysis for a more realistic situation. Unfortunately, this additional piece of effective action is not known in closed form. From another side, the inclusion of non-trivial axion maybe also done. However, in such case only numerical analysis of equations of motion is possible.

Acknowledgments. The work by SDO has been supported in part by the Norwegian Research Council and RFBR grant N99-02-16617. We are extremely grateful to Jan Myrheim for participation at the early stage of this work and helping in preparation of draft of this ms. We also thank Kåre Olaussen and Shinichi Nojiri for useful discussions.

\section{References}

[1] O. Aharony, S. Gubser, J. Maldacena, H. Ooguri and Y. Oz, hep-th/9905111.

[2] I.L. Buchbinder, S.D. Odintsov and I.L. Shapiro, EFFECTIVE ACTION in QUANTUM GRAVITY, IOP Publishing, Bristol and Philadelphia, 1992.

[3] A. Bytsenko, G. Cognola, L. Vanzo and S. Zerbini, Phys. Repts. 266 (1996) 1.

[4] M. Duff, Class. Quan. Grav. 11 (1994) 1387.

[5] R. Reigert, Phys. Lett. B134 (1984) 56;

E.S. Fradkin and A.A. Tseytlin, Phys. Lett. B134 (1984) 187;

I.L. Buchbinder, S.D. Odintsov and I.L. Shapiro, Phys. Lett. B162 (1985) 92;

I. Antoniadis and E. Mottola, Phys. Rev. D45 (1992) 2013.

[6] A. Starobinsky, Phys. Lett. B91 (1980) 99;

S.G. Mamaev and V.M. Mostepanenko, JETP 51 (1980) 9.

[7] M. Kaku, P.K. Townsend, and P. van Nieuwenhuizen, Phys. Rev. D17 (1978) 3179;

E. Bergshoeff, M. de Roo, and B. de Wit, Nucl. Phys. B182 (1981) 173;

E.S. Fradkin and A.A. Tseytlin, Phys. Repts. 119 (1985) 233.

[8] H. Liu and A. Tseytlin, Nucl. Phys. B533 (1998) 88, hep-th 9804083.

[9] I. Brevik and S.D. Odintsov, Phys. Lett. B455 (1999) 104, hep-th/9902418.

[10] G.W. Gibbons, M.B. Green, and M.J. Perry, Phys. Lett. B370 (1996) 37. 